\title{
Existence results for a multipoint boundary value problem of nonlinear sequential Hadamard fractional differential equations
}

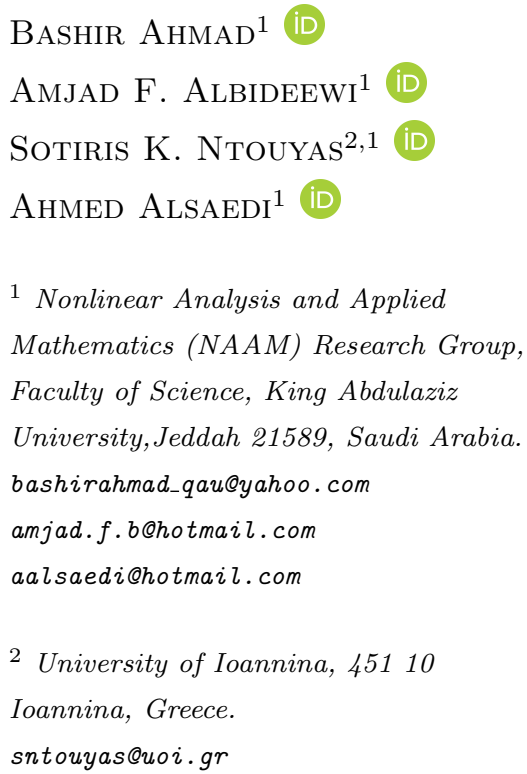

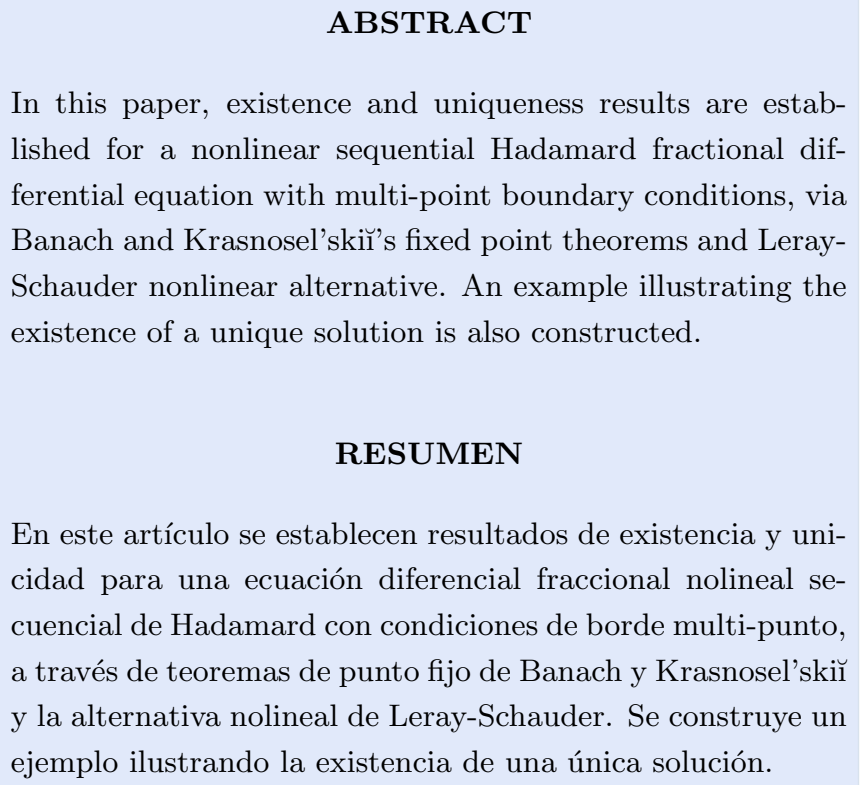

In this paper, existence and uniqueness results are established for a nonlinear sequential Hadamard fractional differential equation with multi-point boundary conditions, via Banach and Krasnosel'skiı̌'s fixed point theorems and LeraySchauder nonlinear alternative. An example illustrating the existence of a unique solution is also constructed.

\section{RESUMEN}

En este artículo se establecen resultados de existencia y unicidad para una ecuación diferencial fraccional nolineal secuencial de Hadamard con condiciones de borde multi-punto, a través de teoremas de punto fijo de Banach y Krasnosel'skiı̌ y la alternativa nolineal de Leray-Schauder. Se construye un ejemplo ilustrando la existencia de una única solución.

Keywords and Phrases: Hadamard fractional integral; Hadamard fractional derivative; multi-point boundary conditions; existence; fixed point theorems.

2020 AMS Mathematics Subject Classification: 34A08, 34A12, 34B15. 


\section{Introduction}

Fractional calculus has been extensively developed during the last few decades as the techniques of this branch of mathematics considerably improved the mathematical modeling of many scientific phenomena, for instance, see [16, 17]. In particular, fractional-order nonlocal boundary value problems are found to be of significant interest for many researchers. Much of the literature on this class of problems is based on Riemann-Liouville or Liouville-Caputo type fractional order differential equations. For details, we refer the reader to some recent works [27] and the references cited therein. In addition to Riemann-Liouville and Caputo type derivatives, there is another kind of derivative, which contains logarithmic function of arbitrary exponent in its definition. This derivative is known as Hadamard derivative [14] and its construction is invariant in relation to dilation and is quite suitable for the problems with semi-infinite domain. For example, Lamb-Bateman integral equation is the one containing Hadamard fractional derivatives of order $1 / 2$ [8]. In [11], a modified Lamb-Bateman equation involving Hadamard derivative and fractional Hyper-Besseltype operators was studied. One can find application details of Hadamard fractional differential equations in the articles $[12,20]$. For some recent results on Hadamard type fractional differential equations, for instance, see $[2,4,5,10,18,19,21,22,23,25,26]$. In a recent monograph [3], one can find a detailed description of initial/boundary value problems and inequalities involving Hadamard fractional differential equations and inclusions. New multiple positive solutions for Hadamard-type fractional differential equations with nonlocal conditions on an infinite interval were studied in [28]. In [6], the authors studied a coupled system of Caputo-Hadamard type sequential fractional differential equations supplemented with nonlocal boundary conditions involving Hadamard fractional integrals. A Caputo-Hadamard fractional turbulent flow model was studied in [24]. However, the Hadamard-type fractional boundary value problems are not sufficiently studied in the mainstream literature.

In this paper, motivated by aforementioned work on Hadamard fractional differential equations, we introduce and study a nonlocal multipoint boundary value problem involving a nonlinear sequential Hadamard fractional differential equation to enrich the related literature. Precisely, we investigate the existence criteria for the following problem:

$$
\left\{\begin{array}{l}
\left({ }^{H} D^{\alpha}+\lambda{ }^{H} D^{\alpha-1}\right) x(t)=f(t, x(t)), \quad 1<\alpha \leq 2, \quad 1<t<T, \\
x(1)=0, \quad x(T)=\sum_{j=1}^{m} \beta_{j} x\left(t_{j}\right),
\end{array}\right.
$$

where ${ }^{H} D^{(\cdot)}$ denotes the Hadamard fractional derivative of order $\alpha, f:[1, T] \times \mathbb{R} \rightarrow \mathbb{R}$ is a continuous function, $\lambda \in \mathbb{R}^{+}, t_{j}, j=1,2, \ldots, m$, are given points with $1 \leq t_{1} \leq \ldots \leq t_{m}<T$, and $\beta_{j}$ are appropriate real numbers. An existence and uniqueness result is proved via Banach's fixed point theorem and also two existence results are established by using Krasnosel'skiǔ's fixed point theorem and Leray-Schauder nonlinear alternative. 
The remaining part of the paper is structured as follows: In Section 2 we recall the related background material and establish a lemma regarding a linear variant of the problem (1.1), useful to transform the problem (1.1) into an equivalent fixed point problem. Section 3 contains the main results for the problem (1.1). An example illustrating the existence and uniqueness result is also included.

\section{Preliminaries}

We introduce notations and definitions of fractional calculus.

Definition 2.1. ([3, 17]) The Hadamard fractional integral of order $q \in \mathbb{C}, \mathfrak{R}(q)>0$, for a function $g \in L^{p}[a, b], 0 \leq a \leq t \leq b \leq \infty$, is defined as

$$
\begin{aligned}
I_{a^{+}}^{q} g(t) & =\frac{1}{\Gamma(q)} \int_{a}^{t}\left(\log \frac{t}{s}\right)^{q-1} \frac{g(s)}{s} d s \\
I_{b^{-}}^{q} g(t) & =\frac{1}{\Gamma(q)} \int_{t}^{b}\left(\log \frac{s}{t}\right)^{q-1} \frac{g(s)}{s} d s .
\end{aligned}
$$

Definition 2.2. ([3, 17]) Let $[a, b] \subset \mathbb{R}, \delta=t \frac{d}{d t}$ and $A C_{\delta}^{n}[a, b]=\left\{g:[a, b] \rightarrow \mathbb{R}: \delta^{n-1}(g(t)) \in\right.$ $A C[a, b]\}$. The Hadamard derivative of fractional order $q$ for a function $g \in A C_{\delta}^{n}[a, b]$ is defined as

$$
\begin{aligned}
& D_{a^{+}}^{q} g(t)=\delta^{n}\left(I_{a^{+}}^{n-q}\right)(t)=\frac{1}{\Gamma(n-q)}\left(t \frac{d}{d t}\right)^{n} \int_{a}^{t}\left(\log \frac{t}{s}\right)^{n-q-1} \frac{g(s)}{s} d s \\
& D_{b^{-}}^{q} g(t)=(-\delta)^{n}\left(I_{b^{-}}^{n-q}\right)(t)=\frac{1}{\Gamma(n-q)}\left(-t \frac{d}{d t}\right)^{n} \int_{t}^{b}\left(\log \frac{s}{t}\right)^{n-q-1} \frac{g(s)}{s} d s
\end{aligned}
$$

where $n-1<q<n, n=[q]+1$ and $[q]$ denotes the integer part of the real number $q$ and $\log (\cdot)=\log _{e}(\cdot)$.

For more details of the Hadamard fractional integrals and derivatives, we refer the reader to Section 2.7 in the text [17].

Lemma 2.3. Let $x \in C_{\delta}^{2}([1, T], \mathbb{R})$ and $g \in C([1, T], \mathbb{R})$. The (integral) solution of the linear Hadamard fractional boundary value problem:

$$
\left\{\begin{array}{l}
\left({ }^{H} D^{\alpha}+\lambda{ }^{H} D^{\alpha-1}\right) x(t)=g(t), \quad 1<\alpha \leq 2, \quad 1<t<T, \\
x(1)=0, \quad x(T)=\sum_{j=1}^{m} \beta_{j} x\left(t_{j}\right),
\end{array}\right.
$$


is given by

$$
\begin{aligned}
x(t)= & \frac{1}{\gamma}\left(t^{-\lambda} \int_{1}^{t} s^{\lambda-1}(\log s)^{\alpha-2} d s\right)\left\{\frac{\sum_{j=1}^{m} \beta_{j} t_{j}^{-\lambda}}{\Gamma(\alpha-1)} \int_{1}^{t_{j}} s^{\lambda-1}\left(\int_{1}^{s}\left(\log \frac{s}{r}\right)^{\alpha-2} \frac{g(r)}{r} d r\right) d s\right. \\
& \left.-\frac{T^{-\lambda}}{\Gamma(\alpha-1)} \int_{1}^{T} s^{\lambda-1}\left(\int_{1}^{s}\left(\log \frac{s}{r}\right)^{\alpha-2} \frac{g(r)}{r} d r\right) d s\right\} \\
& +\frac{t^{-\lambda}}{\Gamma(\alpha-1)} \int_{1}^{t} s^{\lambda-1}\left(\int_{1}^{s}\left(\log \frac{s}{r}\right)^{\alpha-2} \frac{g(r)}{r} d r\right) d s,
\end{aligned}
$$

where it is assumed that

$$
\gamma:=T^{-\lambda} \int_{1}^{T} s^{\lambda-1}(\log s)^{\alpha-2} d s-\sum_{j=1}^{m} \beta_{j} t_{j}^{-\lambda} \int_{1}^{t_{j}} s^{\lambda-1}(\log s)^{\alpha-2} d s \neq 0 .
$$

Proof. The linear Hadamard fractional differential equation in (2.1) can be rewritten as

$$
{ }^{H} D^{\alpha-1}(t D+\lambda) x(t)=g(t), \quad t \in[1, T] .
$$

Applying the Hadamard fractional operator $I^{\alpha-1}$ on both sides of (2.4), we get

$$
\left(D+\frac{\lambda}{t}\right) x(t)=t^{-1}\left(c_{1}(\log t)^{\alpha-2}+I^{\alpha-1} g(t)\right),
$$

which can be rewritten as

$$
D\left(t^{\lambda} x(t)\right)=c_{1} t^{\lambda-1}(\log t)^{\alpha-2}+t^{\lambda-1} I^{\alpha-1} g(t) .
$$

Integrating (2.5) from 1 to $t$, we get

$$
x(t)=c_{0} t^{-\lambda}+c_{1} t^{-\lambda} \int_{1}^{t} s^{\lambda-1}(\log s)^{\alpha-2} d s+t^{-\lambda} \int_{1}^{t} s^{\lambda-1} I^{\alpha-1} g(s) d s,
$$

where $c_{i},(i=0,1)$ are unknown arbitrary constants. Using the initial condition $x(1)=0$ in $(2.6)$ implies that $c_{0}=0$, which leads to

$$
x(t)=c_{1} t^{-\lambda} \int_{1}^{t} s^{\lambda-1}(\log s)^{\alpha-2} d s+t^{-\lambda} \int_{1}^{t} s^{\lambda-1} I^{\alpha-1} g(s) d s .
$$

Now using the condition $x(T)=\sum_{j=1}^{m} \beta_{j} x\left(t_{j}\right)$ in (2.7), we have

$$
\begin{aligned}
& c_{1} T^{-\lambda} \int_{1}^{T} s^{\lambda-1}(\log s)^{\alpha-2} d s+T^{-\lambda} \int_{1}^{T} s^{\lambda-1} I^{\alpha-1} g(s) d s \\
& \quad=c_{1} \sum_{j=1}^{m} \beta_{j} t_{j}^{-\lambda} \int_{1}^{t_{j}} s^{\lambda-1}(\log s)^{\alpha-2} d s+\sum_{j=1}^{m} \beta_{j} t_{j}^{-\lambda} \int_{1}^{t_{j}} s^{\lambda-1} I^{\alpha-1} g(s) d s,
\end{aligned}
$$

which, on solving for $c_{1}$ together with (2.3), yields

$$
c_{1}=\frac{1}{\gamma}\left[\sum_{j=1}^{m} \beta_{j} t_{j}^{-\lambda} \int_{1}^{t_{j}} s^{\lambda-1} I^{\alpha-1} g(s) d s-T^{-\lambda} \int_{1}^{T} s^{\lambda-1} I^{\alpha-1} g(s) d s\right] .
$$

Substituting the above value of $c_{1}$ in (2.7), we get the desired solution (2.2). The converse of the lemma follows by a direct computation. This completes the proof. 
The following lemma contains certain estimates that we need in the sequel.

Lemma 2.4. For $g \in C([1, T], \mathbb{R})$ with $\|g\|=\sup _{t \in[1, T]}|g(t)|$, we have

(i) $\left|t^{-\lambda} \int_{1}^{t} s^{\lambda-1}\left(\int_{1}^{s}\left(\log \frac{s}{r}\right)^{\alpha-2} \frac{g(r)}{r} d r\right) d s\right| \leq \frac{(\log T)^{\alpha}}{\alpha(\alpha-1)}\|g\|$.

(ii) $\left|t^{-\lambda} \int_{1}^{t} s^{\lambda-1}(\log s)^{\alpha-2} d s\right| \leq \frac{(\log T)^{\alpha-1}}{(\alpha-1)}$.

Proof. Note that

$$
\int_{1}^{s}\left(\log \frac{s}{r}\right)^{\alpha-2} \frac{1}{r} d r=\frac{(\log s)^{\alpha-1}}{(\alpha-1)} .
$$

Since $s^{\lambda} \leq t^{\lambda}$ for $1<s<t$, then

$$
\begin{aligned}
& \left|t^{-\lambda} \int_{1}^{t} s^{\lambda-1}\left(\int_{1}^{s}\left(\log \frac{s}{r}\right)^{\alpha-2} \frac{g(r)}{r} d r\right) d s\right| \\
\leq & \sup _{t \in[1, T]}\left|t^{-\lambda} \int_{1}^{t} s^{\lambda-1}\left(\int_{1}^{s}\left(\log \frac{s}{r}\right)^{\alpha-2} \frac{g(r)}{r} d r\right) d s\right| \\
\leq & \|g\| \sup _{t \in[1, T]}\left|t^{-\lambda} \int_{1}^{t} s^{\lambda-1}\left(\frac{(\log s)^{\alpha-1}}{(\alpha-1)}\right) d s\right| \\
\leq & \frac{\|g\|(\log T)^{\alpha}}{\alpha(\alpha-1)}
\end{aligned}
$$

\section{Existence and uniqueness results}

Let $\mathcal{G}=C([1, T], \mathbb{R})$ denote the Banach space of all continuous functions from $[1, T]$ to $\mathbb{R}$ endowed with the usual norm $\|x\|=\sup \{|x(t)|: t \in[1, T]\}$, and $C_{\delta}^{n}([1, T], \mathbb{R})$ denotes the Banach space of all real valued functions $g$ such that $\delta^{n} g \in \mathcal{G}$.

Using Lemma 2.3, we can transform the problem (1.1) into a fixed point problem as $x=\mathcal{P} x$, where the operator $\mathcal{P}: \mathcal{G} \rightarrow \mathcal{G}$ is defined by

$$
\begin{aligned}
(\mathcal{P} x)(t)= & \frac{1}{\gamma}\left(t^{-\lambda} \int_{1}^{t} s^{\lambda-1}(\log s)^{\alpha-2} d s\right) \\
& \times\left\{\frac{\sum_{j=1}^{m} \beta_{j} t_{j}^{-\lambda}}{\Gamma(\alpha-1)} \int_{1}^{t_{j}} s^{\lambda-1}\left(\int_{1}^{s}\left(\log \frac{s}{r}\right)^{\alpha-2} \frac{f(r, x(r))}{r} d r\right) d s\right. \\
& \left.-\frac{T^{-\lambda}}{\Gamma(\alpha-1)} \int_{1}^{T} s^{\lambda-1}\left(\int_{1}^{s}\left(\log \frac{s}{r}\right)^{\alpha-2} \frac{f(r, x(r))}{r} d r\right) d s\right\} \\
& +\frac{t^{-\lambda}}{\Gamma(\alpha-1)} \int_{1}^{t} s^{\lambda-1}\left(\int_{1}^{s}\left(\log \frac{s}{r}\right)^{\alpha-2} \frac{f(r, x(r))}{r} d r\right) d s, \quad t \in[1, T] .
\end{aligned}
$$


For computational convenience, we set

$$
\Lambda=\frac{(\log T)^{\alpha-1}}{|\gamma|(\alpha-1)}\left[\frac{\sum_{j=1}^{m}\left|\beta_{j}\right|(\log T)^{\alpha}}{\Gamma(\alpha+1)}+\frac{(\log T)^{\alpha}}{\Gamma(\alpha+1)}\right]+\frac{(\log T)^{\alpha}}{\Gamma(\alpha+1)} .
$$

In the next theorem, we prove the uniqueness of solutions for problem (1.1) via Banach's fixed point theorem.

Theorem 3.1. Let $f:[1, T] \times \mathbb{R} \rightarrow \mathbb{R}$ be a continuous function and there exists a constant $L>0$ such that:

$\left(H_{1}\right)|f(t, x)-f(t, y)| \leq L|x-y|, \forall t \in[1, T]$ and $x, y \in \mathbb{R}$.

Then, problem (1.1) has a unique solution on $[1, T]$ if $L \Lambda<1$, where $\Lambda$ is given by (3.2).

Proof. Let us define $M$ be finite number given by $M=\sup _{t \in[1, T]}|f(t, 0)|$, and show that $\mathcal{P} B_{r} \subset B_{r}$, where $B_{r}=\{x \in C[1, T]:\|x\| \leq r\}$ with $r \geq \frac{M \Lambda}{1-L \Lambda}$.

For $x \in B_{r}, t \in[1, T]$, using $\left(H_{1}\right)$, we get

$$
\begin{aligned}
|f(t, x(t))| & =|f(t, x(t))-f(t, 0)+f(t, 0)| \\
& \leq|f(t, x(t))-f(t, 0)|+|f(t, 0)| \\
& \leq L\|x\|+M \leq L r+M .
\end{aligned}
$$

Then

$$
\begin{aligned}
|\mathcal{P}(x)(t)| \leq & \sup _{t \in[1, T]}\left\{\frac{1}{|\gamma|}\left(t^{-\lambda} \int_{1}^{t} s^{\lambda-1}(\log s)^{\alpha-2} d s\right)\right. \\
& \times\left[\frac{\sum_{j=1}^{m}\left|\beta_{j} t_{j}^{-\lambda}\right|}{\Gamma(\alpha-1)} \int_{1}^{t_{j}} s^{\lambda-1}\left(\int_{1}^{s}\left(\log \frac{s}{r}\right)^{\alpha-2} \frac{|f(r, x(r))|}{r} d r\right) d s\right. \\
& \left.+\frac{T^{-\lambda}}{\Gamma(\alpha-1)} \int_{1}^{T} s^{\lambda-1}\left(\int_{1}^{s}\left(\log \frac{s}{r}\right)^{\alpha-2} \frac{|f(r, x(r))|}{r} d r\right) d s\right] \\
& \left.+\frac{t^{-\lambda}}{\Gamma(\alpha-1)} \int_{1}^{t} s^{\lambda-1}\left(\int_{1}^{s}\left(\log \frac{s}{r}\right)^{\alpha-2} \frac{|f(r, x(r))|}{r} d r\right) d s\right\} \\
\leq & (L r+M)\left[\frac{(\log T)^{\alpha-1}}{|\gamma|(\alpha-1)}\left(\frac{\sum_{j=1}^{m}\left|\beta_{j}\right|(\log T)^{\alpha}}{\Gamma(\alpha+1)}+\frac{(\log T)^{\alpha}}{\Gamma(\alpha+1)}\right)+\frac{(\log T)^{\alpha}}{\Gamma(\alpha+1)}\right] \\
\leq & \Lambda(L r+M) \leq r .
\end{aligned}
$$

In consequence, $\|\mathcal{P} x\| \leq r$, for any $x \in B_{r}$, which shows that $\mathcal{P} B_{r} \subset B_{r}$.

Now we prove that the operator $\mathcal{P}$ is a contraction. For $(x, y) \in C([1, T], \mathbb{R})$ and for each $t \in[1, T]$, 
we obtain

$$
\begin{aligned}
& |(\mathcal{P} x)(t)-(\mathcal{P} y)(t)| \\
\leq & \sup _{t \in[1, T]}\left\{\frac{1}{|\gamma|}\left(t^{-\lambda} \int_{1}^{t} s^{\lambda-1}(\log s)^{\alpha-2} d s\right)\right. \\
& \times\left[\frac{\sum_{j=1}^{m}\left|\beta_{j} t_{j}^{-\lambda}\right|}{\Gamma(\alpha-1)} \int_{1}^{t_{j}} s^{\lambda-1}\left(\int_{1}^{s}\left(\log \frac{s}{r}\right)^{\alpha-2} \frac{|f(r, x(r))-f(r, y(r))|}{r} d r\right) d s\right. \\
& \left.+\frac{T^{-\lambda}}{\Gamma(\alpha-1)} \int_{1}^{T} s^{\lambda-1}\left(\int_{1}^{s}\left(\log \frac{s}{r}\right)^{\alpha-2} \frac{|f(r, x(r))-f(r, y(r))|}{r} d r\right) d s\right] \\
& \left.+t^{-\lambda} \int_{1}^{t} s^{\lambda-1}\left(\int_{1}^{s}\left(\log \frac{s}{r}\right)^{\alpha-2} \frac{|f(r, x(r))-f(r, y(r))|}{r} d r\right) d s\right\} \\
\leq & L\left[\frac{(\log T)^{\alpha-1}}{|\gamma|(\alpha-1)}\left(\frac{\sum_{j=1}^{m}\left|\beta_{j}\right|(\log T)^{\alpha}}{\Gamma(\alpha+1)}+\frac{(\log T)^{\alpha}}{\Gamma(\alpha+1)}\right)+\frac{(\log T)^{\alpha}}{\Gamma(\alpha+1)}\right] \\
\leq & L \Lambda\|x-y\| .
\end{aligned}
$$

By the given condition $L \Lambda<1$, it follows that the operator $\mathcal{P}$ is a contraction. Thus, the conclusion of the theorem follows by the contraction mapping principle (the Banach fixed point theorem). The proof is complete.

The following existence result is based on the Leray-Schauder nonlinear alternative.

Theorem 3.2 (Nonlinear alternative for single valued maps [13]). Let $E$ be a Banach space, $C$ a closed, convex subset of $E, U$ an open subset of $C$ and $0 \in U$. Suppose that $F: \bar{U} \rightarrow C$ is a continuous, compact (that is, $F(\bar{U})$ is a relatively compact subset of $C$ ) map. Then either

(i) F has a fixed point in $\bar{U}$, or

(ii) there is a $u \in \partial U$ (the boundary of $U$ in $C$ ) and $\nu \in(0,1)$ with $u=\nu F(u)$.

Theorem 3.3. Let $f:[1, T] \times \mathbb{R} \rightarrow \mathbb{R}$ be a continuous function such that the following conditions hold:

$\left(H_{2}\right)$ There exists a function $k \in C\left([1, T], \mathbb{R}^{+}\right)$and a nondecreasing function $\Psi: \mathbb{R}^{+} \rightarrow \mathbb{R}^{+}$such that $|f(t, x)| \leq k(t) \Psi(\|x\|)$ for all $(t, x) \in[1, T] \times \mathbb{R} ;$

$\left(H_{3}\right)$ There exists a positive constant $S>0$ such that

$$
\frac{S}{\Psi(S)\|k\| \Lambda}>1
$$

where $\|k\|=\sup _{t \in[1, T]}|k(t)|$ and $\Lambda$ is defined by (3.2).

Then problem (1.1) has at least one solution on $[1, T]$. 
Proof. Firstly, we shall show that the operator $\mathcal{P}$ defined by (3.1) maps bounded sets into bounded sets in $C([1, T], \mathbb{R})$. For a number $r>0$, let $B_{r}=\{x \in C[1, T]:\|x\| \leq r\}$ be a bounded set in $C([1, T], \mathbb{R})$. Then, by assumption $\left(H_{2}\right)$, we obtain

$$
\begin{aligned}
|(\mathcal{P} x)(t)| \leq & \sup _{t \in[1, T]}\left\{\frac{1}{|\gamma|}\left(t^{-\lambda} \int_{1}^{t} s^{\lambda-1}(\log s)^{\alpha-2} d s\right)\right. \\
& \times\left[\frac{\sum_{j=1}^{m}\left|\beta_{j} t_{j}^{-\lambda}\right|}{\Gamma(\alpha-1)} \int_{1}^{t_{j}} s^{\lambda-1}\left(\int_{1}^{s}\left(\log \frac{s}{r}\right)^{\alpha-2} \frac{|f(r, y(r))|}{r} d r\right) d s\right. \\
& \left.+\frac{T^{-\lambda}}{\Gamma(\alpha-1)} \int_{1}^{T} s^{\lambda-1}\left(\int_{1}^{s}\left(\log \frac{s}{r}\right)^{\alpha-2} \frac{|f(r, x(r))|}{r} d r\right) d s\right] \\
& \left.+\frac{t^{-\lambda}}{\Gamma(\alpha-1)} \int_{1}^{t} s^{\lambda-1}\left(\int_{1}^{s}\left(\log \frac{s}{r}\right)^{\alpha-2} \frac{|f(r, x(r))|}{r} d r\right) d s\right\} \\
\leq & \Psi(\|x\|)\|k\|\left[\frac{(\log T)^{\alpha-1}}{|\gamma|(\alpha-1)}\left(\frac{\sum_{j=1}^{m}\left|\beta_{j}\right|(\log T)^{\alpha}}{\Gamma(\alpha+1)}+\frac{(\log T)^{\alpha}}{\Gamma(\alpha+1)}\right)+\frac{(\log T)^{\alpha}}{\Gamma(\alpha+1)}\right],
\end{aligned}
$$

and consequently,

$$
\|\mathcal{P} x\| \leq \Lambda \Psi(r)\|k\|
$$

Next we show that $\mathcal{P}$ maps bounded sets into equicontinuous sets of $C([1, T], \mathbb{R})$. Let $\tau_{1}, \tau_{2} \in[1, T]$ with $\tau_{1}<\tau_{2}$ and $x \in B_{r}$. Then, we have

$$
\begin{aligned}
\left|(\mathcal{P} x)\left(\tau_{2}\right)-(\mathcal{P} x)\left(\tau_{1}\right)\right| \leq & \Psi(r)\|k\|\left\{\frac { 1 } { | \gamma | } \left(\left|\tau_{1}^{-\lambda}-\tau_{2}^{-\lambda}\right| \int_{1}^{\tau_{1}} s^{\lambda-1}(\log s)^{\alpha-2} d s\right.\right. \\
& \left.+\tau_{2}^{-\lambda} \int_{\tau_{1}}^{\tau_{2}} s^{\lambda-1}(\log s)^{\alpha-2} d s\right) \\
& \times\left[\frac{\sum_{j=1}^{m}\left|\beta_{j}\right|\left|t_{j}^{-\lambda}\right|}{\Gamma(\alpha-1)} \int_{1}^{\tau_{1}} s^{\lambda-1}\left(\int_{1}^{s}\left(\log \frac{s}{r}\right)^{\alpha-2} \frac{1}{r} d r\right) d s\right. \\
& \left.+\frac{T^{-\lambda}}{\Gamma(\alpha-1)} \int_{1}^{T} s^{\lambda-1}\left(\int_{1}^{s}\left(\log \frac{s}{r}\right)^{\alpha-2} \frac{1}{r} d r\right) d s\right] \\
& +\frac{\left|\tau_{1}^{-\lambda}-\tau_{2}^{-\lambda}\right|}{\Gamma(\alpha-1)} \int_{1}^{\tau_{1}} s^{\lambda-1}\left(\int_{1}^{s}\left(\log \frac{s}{r}\right)^{\alpha-2} \frac{1}{r} d r\right) d s \\
& +\frac{\tau_{2}^{-\lambda}}{\Gamma(\alpha-1)} \int_{\tau_{1}}^{\tau_{2}} s^{\lambda-1}\left(\int_{1}^{s}\left(\log \frac{s}{r}\right)^{\alpha-2} \frac{1}{r} d r\right) d s .
\end{aligned}
$$

Obviously the right-hand side of the above inequality tends to zero independently of $x \in B_{r}$ as $\tau_{2}-\tau_{1} \rightarrow 0$. Therefore, by the Arzelá-Ascoli Theorem, the operator is completely continuous.

The result will follow from Theorem 3.2 once it is established that the set of all solutions to equations $x=\nu \mathcal{P} x$ for $\nu \in(0,1)$ is bounded. Let $x$ be a solution of problem (1.1). Then, for $t \in[1, T]$, as in the first step, we can find that

$$
\|x\|=\sup _{t \in[1, T]}\{\nu(\mathcal{P} x)(t)\} \leq \Lambda \Psi(\|x\|)\|k\|,
$$


which leads to

$$
\frac{\|x\|}{\Lambda \Psi(\|x\|)\|k\|} \leq 1
$$

By condition $\left(H_{3}\right)$, there exists $S>0$ such that $\|x\| \neq S$. Let us set $U=\{x \in C([1, T], \mathbb{R}):\|x\|<$ $S\}$. Note that the operator $\mathcal{P}: \bar{U} \rightarrow C([1, T], \mathbb{R})$ is continuous and completely continuous. From the choice of $U$, there is no $x \in \partial U$ such that $x=\nu \mathcal{P} x$ for some $\nu \in(0,1)$. Consequently, we deduce by Theorem 3.2 that $\mathcal{P}$ has a fixed point $x \in \bar{U}$, which is a solution of problem (1.1). This completes the proof.

Our final existence result is based on Krasnosel'skiı̌'s fixed point theorem.

Theorem 3.4. (Krasnosel'skil's fixed point theorem) Let $M$ be a closed convex and nonempty subset of a Banach space $X$. Let $A, B$ be the operators such that

(i) $A x+B y \in M$ whenever $x, y \in M$,

(ii) $B$ is a contraction mapping,

(iii) A is compact and continuous.

Then there exists $z \in M$ such that $z=A z+B z$.

Theorem 3.5. Let $f:[1, T] \times \mathbb{R} \rightarrow \mathbb{R}$ be a continuous function satisfying the condition $\left(H_{1}\right)$. In addition, we assume that:

$\left(H_{4}\right)|f(t, x)| \leq \mu(t)$ for all $(t, x) \in[1, T] \times \mathbb{R}, \mu \in C\left([1, T], \mathbb{R}^{+}\right)$.

Then, the boundary value problem (1.1) has at least one solution on $[1, T]$, provided that

$$
L\left(\Lambda-\frac{(\log T)^{\alpha}}{\Gamma(\alpha+1)}\right)<1
$$

where $\Lambda$ is given by (3.2).

Proof. Consider $B_{\rho}=\{x \in \mathcal{G}:\|x\| \leq \rho\},\|\mu\|=\sup _{t \in[0,1]}|\mu(t)|$, with $\rho \geq\|\mu\| \Lambda$. Then we define the operators $\mathcal{P}_{1}$ and $\mathcal{P}_{2}$ on $B_{\rho}$ as

$$
\begin{aligned}
\left(\mathcal{P}_{1} x\right)(t)= & \frac{1}{\gamma}\left(t^{-\lambda} \int_{1}^{t} s^{\lambda-1}(\log s)^{\alpha-2} d s\right) \\
& \times\left\{\frac{\sum_{j=1}^{m} \beta_{j} t_{j}^{-\lambda}}{\Gamma(\alpha-1)} \int_{1}^{t_{j}} s^{\lambda-1}\left(\int_{1}^{s}\left(\log \frac{s}{r}\right)^{\alpha-2} \frac{f(r, x(r))}{r} d r\right) d s\right. \\
& \left.-\frac{T^{-\lambda}}{\Gamma(\alpha-1)} \int_{1}^{T} s^{\lambda-1}\left(\int_{1}^{s}\left(\log \frac{s}{r}\right)^{\alpha-2} \frac{f(r, x(r))}{r} d r\right) d s\right\}, \quad t \in[1, T], \\
\left(\mathcal{P}_{2} x\right)(t)= & \frac{t^{-\lambda}}{\Gamma(\alpha-1)} \int_{1}^{t} s^{\lambda-1}\left(\int_{1}^{s}\left(\log \frac{s}{r}\right)^{\alpha-2} \frac{f(r, x(r))}{r} d r\right) d s, \quad t \in[1, T] .
\end{aligned}
$$


As in 3.1 we can prove that $\left\|\mathcal{P}_{1} x+\mathcal{P}_{2} y\right\| \leq\|\mu\| \Lambda<\rho$, and thus, $\mathcal{P}_{1} x+\mathcal{P}_{2} y \in B_{\rho}$. By using condition (3.3) it is easy to prove that $\mathcal{P}_{1}$ is a contraction (see also 3.1). Moreover the continuous operator $\mathcal{P}_{2}$ is uniformly bounded, as

$$
\left\|\mathcal{P}_{2}\right\| \leq \frac{(\log T)^{\alpha}}{\Gamma(\alpha+1)}\|\mu\|
$$

and equicontinuous as

$$
\begin{aligned}
\left|\left(\mathcal{P}_{2} x\right)\left(\tau_{2}\right)-\left(\mathcal{P}_{2} x\right)\left(\tau_{1}\right)\right| \leq & \frac{\left|\tau_{1}^{-\lambda}-\tau_{2}^{-\lambda}\right|}{\Gamma(\alpha-1)} \int_{1}^{\tau_{1}} s^{\lambda-1}\left(\int_{1}^{s}\left(\log \frac{s}{r}\right)^{\alpha-2} \frac{1}{r} d r\right) d s \\
& +\frac{\tau_{2}^{-\lambda}}{\Gamma(\alpha-1)} \int_{\tau_{1}}^{\tau_{2}} s^{\lambda-1}\left(\int_{1}^{s}\left(\log \frac{s}{r}\right)^{\alpha-2} \frac{1}{r} d r\right) d s .
\end{aligned}
$$

Hence, by Arzelá-Ascoli Theorem, $\mathcal{P}_{2}$ is compact on $B_{\rho}$. Thus all the assumptions of 3.4 are satisfied and the conclusion of 3.4 implies that the boundary value problem (1.1) has at least one solution on $[1, T]$. The proof is completed.

Example 3.6. Consider the boundary value problem for Hadamard fractional differential equations

$$
\left\{\begin{array}{l}
\left({ }^{H} D^{7 / 4}+2{ }^{H} D^{3 / 4}\right) x(t)=f(t, x(t)), \quad t \in[1, e], \\
x(1)=0, \quad x(e)=\sum_{j=1}^{3} \beta_{j} x\left(t_{j}\right) .
\end{array}\right.
$$

Here, $\alpha=7 / 4, \lambda=2, T=e, m=3, \beta_{1}=1 / 3, \beta_{2}=1 / 9, \beta_{3}=1 / 27, t_{1}=5 / 4, t_{2}=3 / 2, t_{3}=$ $7 / 4$ and $f(t, x)=\frac{1}{13 \sqrt{t^{2}+24}} \frac{|x|}{1+|x|}+\frac{1}{t+2}+\log t$.

Clearly, $L=1 / 65$ as $|f(t, x)-f(t, y)| \leq(1 / 65)|x-y|$. Using the given data, we have $|\gamma| \approx 0.691358$ and $\Lambda \approx 1.104500$. Then $L \Lambda \approx 0.016992<1$. Thus, by 3.1, the boundary value problem (3.4) has a unique solution on $[1, e]$.

\section{Acknowledgement}

The authors thank the reviewers for their useful comments on our work that led to the improvement of the original manuscript. 


\section{References}

[1] B. Ahmad and S. K. Ntouyas, "Some fractional-order one-dimensional semi-linear problems under nonlocal integral boundary conditions", Rev. R. Acad. Cienc. Exactas, Fís. Nat. Ser. A Mat. RACSAM, vol. 110, no. 1, pp. 159-172, 2016.

[2] B. Ahmad and S.K. Ntouyas, "A fully Hadamard type integral boundary value problem of a coupled system of fractional differential equations", Fract. Calc. Appl. Anal., vol. 17, no. 2, pp. 348-360, 2014.

[3] B. Ahmad, A. Alsaedi, S. K. Ntouyas and J. Tariboon, Hadamard-type fractional differential equations, inclusions and inequalities, Cham, Switzerland: Springer, 2017.

[4] S. Aljoudi, B. Ahmad, J.J. Nieto and A. Alsaedi, "A coupled system of Hadamard type sequential fractional differential equations with coupled strip conditions", Chaos Solitons Fractals, vol. 91, pp. 39-46, 2016.

[5] S. Aljoudi, B. Ahmad, J.J. Nieto and A. Alsaedi, "On coupled Hadamard type sequential fractional differential equations with variable coefficients and nonlocal integral boundary conditions", Filomat, vol. 31, no. 19, pp. 6041-6049, 2017.

[6] S. Aljoudi, B. Ahmad and A. Alsaedi, "Existence and uniqueness results for a coupled system of Caputo-Hadamard fractional differential equations with nonlocal Hadamard type integral boundary conditions", Fractal Fract. vol. 4, no. 13, 15 pages, 2020.

[7] I. Area, J. Losada and J.J. Nieto, "A note on the fractional logistic equation", Phys. A, vol. 444, pp. 182-187, 2016.

[8] D. Babusci, G. Dattoli and D. Sacchetti, "The Lamb-Bateman integral equation and the fractional derivatives", Fract. Calc. Appl. Anal., vol. 14, pp. 317-320, 2011.

[9] Y. Ding, Z. Wei, J. Xu and D. O'Regan, "Extremal solutions for nonlinear fractional boundary value problems with p-Laplacian", J. Comput. Appl. Math., vol. 288, pp. 151-158, 2015.

[10] X. Du, Y. Meng and H. Pang, "Iterative positive solutions to a coupled Hadamard-type fractional differential system on infinite domain with the multistrip and multipoint mixed boundary conditions", J. of Funct. Spaces, Art. ID 6508075, 16 pages, 2020.

[11] R. Garra and F. Polito, "On some operators involving Hadamard derivatives", Integral Transforms Spec. Funct., vol. 24, no. 10, pp. 773-782, 2013.

[12] R. Garra, E. Orsingher and F. Polito, "A note on Hadamard fractional differential equations with varying coefficients and their applications in probability", Mathematics, vol. 6 , no. 4,10 pages, 2018. 
[13] A. Granas and J. Dugundji, Fixed Point Theory, Springer Monogr. in Math., New York: Springer-Verlag, 2003.

[14] J. Hadamard, "Essai sur l'étude des fonctions données par leur developpment de Taylor", J. de Math. Pures Appl., vol. 8, pp. 101-186, 1892.

[15] J. Henderson and N. Kosmatov, "Eigenvalue comparison for fractional boundary value problems with the Caputo derivative", Fract. Calc. Appl. Anal., vol. 17, pp. 872-880, 2014.

[16] A. A. Kilbas, H. M. Srivastava and J. J. Trujillo, Theory and Applications of Fractional Differential Equations, North-Holland Mathematics Studies, vol. 204., Amsterdam: Elsevier Science B.V., 2006.

[17] J. Klafter, S. C Lim and R. Metzler (Editors), Fractional Dynamics in Physics, Singapore: World Scientific, 2011.

[18] L. Ma, "On the kinetics of Hadamard-type fractional differential systems", Fract. Calc. Appl. Anal., vol. 23, pp. 553-570, 2020.

[19] Q. Ma, R. Wang, J. Wang and Y. Ma, "Qualitative analysis for solutions of a certain more generalized two-dimensional fractional differential system with Hadamard derivative", Appl. Math. Comput., vol. 257, pp. 436-445, 2015.

[20] R. K. Saxena, R. Garra and E. Orsingher, "Analytical solution of space-time fractional telegraph-type equations involving Hilfer and Hadamard derivatives", Integral Transforms Spec. Funct., vol. 27, no. 1, pp. 30-42, 2016.

[21] J. Tariboon, S. K. Ntouyas, S. Asawasamrit and C. Promsakon, "Positive solutions for Hadamard differential systems with fractional integral conditions on an unbounded domain", Open Math., vol. 15, no. 1, pp. 645-666, 2017.

[22] J. R. Wang, Y. Zhou and M. Medved, "Existence and stability of fractional differential equations with Hadamard derivative", Topol. Methods Nonlinear Anal., vol. 41, no 1, pp. 113-133, 2013.

[23] J. R. Wang and Y. Zhang, "On the concept and existence of solutions for fractional impulsive systems with Hadamard derivatives", Appl. Math. Lett., vol. 39, pp. 85-90, 2015.

[24] G. Wang, X. Ren, L. Zhang and B. Ahmad, "Explicit iteration and unique positive solution for a Caputo-Hadamard fractional turbulent flow model", IEEE Access, vol. 7, pp. 109833109839, 2019.

[25] W. Yukunthorn, B. Ahmad, S. K. Ntouyas and J. Tariboon, "On Caputo-Hadamard type fractional impulsive hybrid systems with nonlinear fractional integral conditions", Nonlinear Anal. Hybrid Syst., vol. 19, pp. 77-92, 2016. 
[26] C. Zhai, W. Wang and H. Li, "A uniqueness method to a new Hadamard fractional differential system with four-point boundary conditions", J. Inequal. Appl., Paper No. 207, 16 pages, 2018.

[27] C. Zhai and L. Xu, "Properties of positive solutions to a class of four-point boundary value problem of Caputo fractional differential equations with a parameter", Commun. Nonlinear Sci. Numer. Simul., vol. 19, pp. 2820-2827, 2014.

[28] W. Zhang and J. Ni, "New multiple positive solutions for Hadamard-type fractional differential equations with nonlocal conditions on an infinite interval", Appl. Math. Lett., vol. 118, ID 107165, 10 pages, 2021. 